REVISTA DE DERECHO UNED, NÚM. 17, 2015

\title{
EL PRETENDIDO DERECHO DE AUTODETERMINACIÓN DE LOS POBLADORES DE LAS ISLAS MALVINAS: APUNTES EN TORNO AL REFERÉNDUM DEL AÑO 2013
}

\author{
THE PRETENDED SELF-DETERMINATION RIGHT \\ OF THE ISLAS MALVINAS RESIDENTS: NOTES ABOUT \\ THE 2013 REFERENDUM
}

Gonzalo Gabriel CARRAnZA

Resumen: Desde hace muchos años existe una disputa soberana entre Argentina y Gran Bretaña por las Islas Malvinas, lo que ha llevado a acciones diplomáticas e, incluso, a la guerra. En los últimos años, los habitantes de las Islas han mostrado su interés por formar parte de Inglaterra como ciudadanos plenos pero, casi al mismo tiempo, han pretendido autodeterminarse como pueblo.

En el presente trabajo analizaremos este propósito autodeterminante de los habitantes de las Islas a la luz del derecho y de la historia que los relaciona con los dos países que disputan su soberanía, sobre todo desde el último referéndum que plantearon en el 2013.

Palabras claves: Islas Malvinas, Autodeterminación, Referéndum, Soberanía, Pueblo.

Abstract: For a long time there is a sovereignty dispute between Argentina and Great Britain because of the «Islas Malvinas», which carried to diplomatic acctions and also a war. During the last years, the island's inhabitants showed their interest of being a part of England as full citizens but, at the same time, they intented the self-determination like a people. 
In this paper we analyze this purpose of the inhabitants from the law perspective and the history that relates the two countries who contest its sovereignty, above all since the last referendum that take place in 2013.

Keywords: Islas Malvinas, Self-determination, Referendum, Sovereignty, People.

Recepción original: 16/06/2015

Aceptación original: 14/07/2015

Sumario: I. Introducción. II. Las Islas Malvinas. A. Su enclave estratégico. B. Breve historia de su descubrimiento y ocupación. C. Los reclamos soberanos argentinos ante los organismos internacionales. D. La Guerra de Malvinas. III. Los ocupadores de las Islas. A. Los kelpers. B. El gobierno de las Islas Malvinas (Falkland Islands). IV. El pretendido derecho de autodeterminación de los kelpers y el referéndum de 2013. A. Nación, pueblo y autodeterminación. 1. ¿Son los kelpers una nación? 2. ¿Pueden los kelpers autodeterminarse como pueblo? B. El referéndum de 2013. 1. El meollo del asunto. 2. El desarrollo del referéndum. 3. La validez del referéndum. El rechazo argentino. 5. Conclusiones.

\section{INTRODUCCIÓN}

Cuando se habla de disputas coloniales, lo primero que se nos viene a la cabeza es la situación vivida en el globo a lo largo de los Siglos XIII a XVIII, quizás en una posición un tanto inocente. Si comparamos hoy los mapas que algunos países de Europa y otros de América dibujan en sus Institutos Cartográficos, podremos ver que algunos territorios aún continúan en disputas coloniales, como lo demuestran las diferencias nominativas de algunos espacios, como islas y archipiélagos.

Dentro de las grandes disputas soberanas que los contemporáneos apreciamos, una de las de mayor relieve es la de las Islas Malvinas ${ }^{1}$, que nuclea en conflicto a Argentina y Gran Bretaña. En ese enclave territorial sureño se ha desarrollado una guerra hace poco más de 30 años que mantuvo en vilo al mundo occidental, sobre todo por el enfrentamiento político, la naturaleza histórica y las consecuencias que tuvo en los dos hemisferios.

${ }^{1}$ La denominación británica es de Falkland Islands. Por razones de identidad, utilizaremos la nominación Islas Malvinas. 
En esas Islas viven desde antaño un grupo de pobladores implantados que han tratado de mantener costumbres británicas y, sobre todo, sostener la posesión de éstas para defenderlas como suyas frente a la Comunidad Internacional, donde se enclavan los debates tendientes a lograr la solución del conflicto, escuchando los argumentos de títulos de derecho que ambas partes poseen.

En este trabajo, pretendemos realizar un acercamiento al recorrido histórico que ha sufrido la posesión isleña junto a una comprensión de las claves que tiene el territorio, para poder así comprender cómo el devenir de los días y sus sucesos ha influido en la población actual. De esta manera, teniendo instrumentos históricos, jurídicos y sociales, podremos acercarnos al entendimiento del referéndum que se realizó en las Islas en el año 2013 en torno al pretendido derecho de autodeterminación de los kelpers, cuestión que nos abrirá las puertas para poder realizar algunas afirmaciones en torno a la disputa soberana, a los derechos puestos en tela de juicio y al futuro diplomático del archipiélago.

\section{LAS ISLAS MALVINAS}

Entender la cuestión Malvinas y adentrarnos en el proceso de referéndum que quisieron llevar a cabo sus pobladores requiere un primer esfuerzo de comprender qué son este conjunto de Islas situadas al sur del Atlántico. Por ello, no sólo es importante atender a su enclave estratégico, sino a las disputas soberanas que se enmarcan en torno a ellas. A continuación, trataremos de adentrarnos brevemente en algunas de las cuestiones esenciales para comprender la dinámica que llevó a Argentina y a Gran Bretaña a encontrarse en disputa actualmente.

\section{A. Su enclave estratégico}

Hay datos que llevan a pensar en que deben existir razones superiores a las objetivas las que levan a Gran Bretaña a querer mantener su disputa sobre las Islas. Es un dato tangible la distancia de 14000 kilómetros entre las Islas y aquel país, como así también el que cuentan con una población 2932 habitantes $^{2}$. Quizás lo que mantiene el dominio colonial es algo bien sencillo: las Islas tienen una posición estratégica en el globo.

${ }^{2}$ Cfr. Documento Falkland Islands Census 2012: headline results, disponible en http://www.falklands.gov.fk/assets/Headline-Results-from-Census-2012.pdf

(C) UNED. Revista de Derecho UNED, núm. 17, 2015 
Las Malvinas se ubican al sur del Océano Atlántico, y son un archipiélago del Mar Argentino, con una distancia de $356.4 \mathrm{~km}$ con la costa del país rioplatense. Esta formación de islas en las que se destacan Soledad y Malvinas, se conforma además por una serie de pequeñas islas e islotes satélites que configuran un nutrido espacio adyacente al territorio del país del sur.

La ubicación denota que éstas son un testigo frecuente del paso de buques que quieren cruzar el Atlántico por el Estrecho de Magallanes. Esta ubicación de preferencia es un punto estratégico para el control de los navíos de carga y pesqueros. Desde 1994, el Estrecho permite que se atraviese el Continente sin tener que viajar hasta el punto más austral, con los peligros que las aguas frías conllevan para las máquinas y tripulaciones, de manera tal que el Estrecho permite evitar el Cabo de Hornos.

Su cercanía con los grandes yacimientos petrolíferos del sur hacen que la relación de las Islas con las cuestiones económicas de notable trascendencia para el mundo entero tengan una destacada preponderancia $^{3}$. Gran Bretaña ha instalado pozos petrolíferos marítimos que exploran en búsqueda de oro negro, con un incalculable valor en el caso de encontrarlos.

La proximidad a la Antártida es otra de las causas. Este espacio de hielo tiene la característica de ser un lugar disputado por numerosas naciones que reclaman parte del territorio en virtud de la proximidad geográfica o la historia, más allá de lo establecido en el Tratado de la Antártida de 1961. Las reservas de agua potable que representa esta masa de hielo permite apreciar con ojos visionarios al mantenimiento de la población en las Islas.

Vemos de esta manera que el sostenimiento de las pretensiones soberanas de Gran Bretaña no sólo se dan por cuestiones que se refieren a particularidades históricas, sino que además existen razones que dan peso al asentamiento de capital humano y al fomento económico del espacio.

\section{B. Breve historia de su descubrimiento y ocupación}

El tema del descubrimiento y ocupación de las Islas «ha sido uno de los temas más intensamente debatidos» ${ }^{4}$ en relación a la disputa. En

${ }^{3}$ Para ampliar, recomiendo leer VILARÓ, Ramón (2007). «En Malvinas huele a petróleo» en Revista Cambio 16, N. ${ }^{\circ} 1845$ (ABR 17), Madrid, págs. 34-35.

${ }^{4}$ Cfr. HOPE, Adrián F. J. (1983). «Soberanía y descolonización de las Islas Malvinas (Falkland Islands)» en Boston College International and comparative law review Vol. 6, N. ${ }^{\circ}$ 2. Boston, Boston College. Pág. 394. 
un artículo coetáneo a la guerra de las Malvinas, Raimundo Díaz Alejo realiza un análisis de la historia de los hechos que llevaron a la ocupación británica de las Islas ${ }^{5}$. De ello, podemos identificar algunas cuestiones trascendentales que relatamos a continuación.

Por un lado, algunos atribuyen a Américo Vespucio el descubrimiento en el año 1501. De sus diarios se lee: «en medio de la tormenta avistamos el 17 de abril una nueva tierra, de la cual recorrimos cerca de veinte leguas, encontrando la costa brava, y no vimos en ella puerto alguno, ni gente, creo que porque el frío era tan intenso que ninguno de la flota podía remediar ni soportarlo» ${ }^{6}$.

Otros atribuyen el descubrimiento de las Islas «al español Pedro de Vera (...), o al portugués Alonso de Camargo, en 1525 y 1539 respectivamente. No dejan los británicos de adjudicar el descubrimiento a John Davis (1592) y a Richard Hawkins (1594), ambos piratas (...), pero ni uno ni otro fijaron latitud ni dieron nombre a las islas, si bien el último las denominó 'Maidenland'»?.

Más allá de los debates, es incuestionable que la primer visita fue en 1600 por el holandés Sebald de Weer «a fin de abastecerse de agua, en el navio 'Geloof', bautizándolas con el nombre de Islas Sebaldinas, que conservan en la toponimia cartográfica de los siglos XVII y XVIII». Luego de ello, el 28 de enero de 1690, John Strong (navegante inglés) atraviesa el Estrecho de San Carlos denominando a las Islas «Falkland» en honor a su protector, Lord Falkland, pasando luego a denominarse de esa forma a todo el conjunto de islas, islas menores e islotes del archipiélago.

Más tarde, serán los franceses los que pasen por allí y se atribuyan su soberanía. Gracias a la «Compañía del Mar del Sur», comienzan a explorar las Islas en distintos viajes y será Luis Antonio de Bougainville el que fundase la primer Colonia, llegando al archipiélago el 31 de Enero de 1764. Comenzó así el asentamiento francés, cuyos pobladores nominaron a las Islas «Malouinas» ${ }^{9}$. El hecho de la ocupación dio lugar al reclamo español, lo que dio lugar al reconocimiento del derecho de España sobre las Islas.

Dieciocho años después, Gran Bretaña volvió a invadirlas en Puerto Egmont, cuestión que llegó al conocimiento Español, que intentó

${ }^{5}$ Cfr. DÍAZ ALEJO, Raimundo (1982). «El problema de las Malvinas» en Quinto Centenario N. . 4, Madrid UCM. Págs.. 175-208.

${ }^{6}$ Cfr. DÍAZ ALEJO, Raimundo (1982). Op. cit. Pág. 176.

${ }^{7}$ Idem.

${ }^{8}$ Cfr. DÍAZ ALEJO, Raimundo (1982). Op. cit. Pág. 179.

9 De allí provendrá, luego, el nombre español de Islas Malvinas. 
la evacuación de la ocupación del archipiélago, resultando victorioso de la empresa.

Con la Independencia de las Provincias Unidas del Río de la Plata, se trasladan los derechos posesorios sobre las Islas al nuevo Estado que se estaba formando, lo que comienza a afirmarse con el envío de una fragata para la toma de posesión. La inminente República Argentina se transforma así en la legítima propietaria de las Islas.

En medio de la convulsión de la organización nacional, el 3 de Enero de 1833 un buque inglés desembarca en las Islas bajo el mando de John James Onslow, comunicando que se reafirmaba la soberanía británica en nombre del Rey. En esa oportunidad, Inglaterra «usurpó las Malvinas en plena paz, pues estaba en vigencia el tratado de Amistad, Comercio y Navegación de 1825, entre ambos países» ${ }^{10}$. El capitán argentino José María Pinedo no se consideró en condiciones de resistir y optó por embarcar a los hombres y retornar a Argentina. Al día siguiente, los británicos arriaron el pabellón argentino, izaron el británico y tomaron posesión de las Islas. Unos años después, en 1835, un grupo de ocho argentinos -encabezados por el gaucho Antonio Rivero- se sublevaron y se trasladaron a las Islas, atacaron la comandancia, dieron muerte a cinco británicos, arriaron la bandera inglesa y pusieron en alto la argentina, respetando a mujeres y niños, gobernando seis meses las Islas «sin que en Buenos Aires se tuviera noticia de lo acontecido» ${ }^{11}$. A partir de la caída de Rivero, continuará el ilegítimo asentamiento de los Kelpers en las Islas Malvinas.

\section{Los reclamos soberanos argentinos ante los organismos internacionales.}

Desde 1833, Argentina reclama las Islas. Se realizó primero un pedido ante el Parlamento Británico, el que fue desoído ${ }^{12}$. Las relaciones diplomáticas desde que Argentina se constituyó como Estado en 1853 y hasta el día de hoy, manifiestan un constante reclamo por parte de la República ante la Corona, hecho que no sólo se ha realizado por manifestaciones públicas, sino que ha sido llevado por los canales correspondientes a través de los Cancilleres y Presidentes argentinos, y además ha encontrado una voz que resuena en las Asambleas Generales

${ }^{10}$ Cfr. DESTEFANI, Laurio H. (1982). Malvinas, Georgias y Sandwich del Sur, ante el conflicto con Gran Bretaña, Buenos Aires, Edipress S. A.. Pág. 7.

${ }^{11}$ Cfr. DÍAZ ALEJO, Raimundo (1982). Op. cit. Pág. 190.

12 Aunque cabe destacar que un británico, William Mollesworth, instó a la devolución de las Islas. 
de la Organización de las Naciones Unidas, en la Organización de los Estados Americanos ${ }^{13}$ y en todo tipo de relación bilateral. La más amplia gama de sectores de ideología política de argentina manifiestan el reclamo y lo hacen suyo, siendo una cuestión nacional al día de hoy.

Cuando Argentina suscribió la Carta de San Francisco en 1945, hizo especial reserva de su soberanía sobre las Islas. La Resolución 1514 de la Asamblea General de las Naciones Unidas ${ }^{14}$ llevó a que el organismo se preocupe por la descolonización, creándose el Comité 24. Bien señala Hope al destacar que «con la creación de las Naciones Unidas después de la Segunda Guerra Mundial, el conflicto anglo-argentino por las Malvinas se convirtió en algo más que una disputa territorial convencional que afecta los intereses puramente bilaterales de dos estados con respecto a una porción de territorio» ${ }^{15}$.

El 16 de diciembre de 1965, se aprobó la Resolución 2065 de la Asamblea General, la cual recita en su artículo 1..$^{\circ}$ que «invita a los Gobiernos de la Argentina y del Reino Unido de Gran Bretaña e Irlanda del Norte a proseguir sin demora las negociaciones recomendadas por el Comité Especial encargado de examinar la situación con respecto a la aplicación de la Declaración sobre la concesión de la independencia a los países y pueblos coloniales a fin de encontrar una solución pacífica al problema, teniendo debidamente en cuenta las disposiciones y los objetivos de la Carta de las Naciones Unidas y de la Resolución 1514 (XV) de la Asamblea General, asi como los intereses de la población de las Islas Malvinas (Falkland Islands)» ${ }^{16}$.

Sobre esta Resolución, cabe destacar el alegato del Embajador Argentino José María Ruda ${ }^{17}$, quien expuso «los hechos históricos que dan razón de la ocupación del territorio nacional argentino probando que la posición británica, que constituye un anacronismo propio de los grandes imperios coloniales, se afirma solamente en la fuerza» ${ }^{18}$. Destacables son las palabras del propio Embajador, cuando señala que «nuestro propósito es persuadir a la comunidad internacional de que las mencionadas Islas son parte integrante del territorio argentino y que el deber jurídico y

${ }^{13}$ Señala Adrián F. J. Hope al respecto que «mediante la acción de la Argentina, el conflicto se convirtió en una cuestión de interés regional dentro de la Organización de Estados Americanos», para ello Cfr. HOPE, Adrián F. J. (1983). Op. cit. Pág. 436.

${ }^{14}$ Del 14 de Diciembre de 1960.

${ }^{15}$ Cfr. HOPE, Adrián F. J. (1983). Op. cit. Pág. 436.

${ }^{16} C f r$. artículo 1. ${ }^{\circ}$, Resolución 2065 de la Asamblea General de la Organización de las Naciones Unidas.

${ }^{17}$ Embajador durante el gobierno del Presidente Arturo Humberto Illia.

${ }_{18}$ Cfr. Documento Soberanía Argentina en Malvinas. A 50 años del "Alegato Ruda» (2014), Buenos Aires, Ministerio de Relaciones Exteriores. Pág. 11. 
moral de Gran Bretaña es devolverlas a su verdadero dueño, afirmándose asi el principio de la soberanía y de la integridad territorial de los Estados, base de las relaciones internacionales pacíficas» ${ }^{19}$.

El 14 de diciembre de 1973, ocho años después de la Resolución 2065, la ONU se manifiesta con la Resolución 3160 de la Asamblea General, diciendo que se encontraba "gravemente preocupada por el hecho de que han transcurrido ocho años desde la adopción de la resolución 2065 (XX) sin que se hayan producido progresos sustanciales en las negociaciones ${ }^{20}$, e insta a ambos gobiernos a proseguir con las negociaciones al respecto.

Desde aquel entonces, Argentina ha sostenido año tras año su legítima defensa soberana sobre las Islas, desconociendo Gran Bretaña dicha afirmación y negándose a llevar adelante el diálogo solicitado por el más alto organismo internacional.

Durante el gobierno de los Kirchner, Argentina ha vuelto a realizar reclamos continuados y de contundencia en las Asambleas Generales de la ONU para que el gobierno de Gran Bretaña atienda el reclamo soberano, siendo uno de los estandartes de los tres mandatos presidenciales del matrimonio que respetan y alientan tanto los sectores aliados como opositores al gobierno.

La causa Malvinas es atendida por los 40 millones de argentinos, quienes se han convertido en una tribuna que clama por su perla austral. La causa Malvinas es una señal identitaria del nacionalismo del sur del continente americano. Dicho por un argentino, "si Malvinas tiene un excepcional valor simbólico, es porque la forma en que miramos las islas es una forma poderosa -nos es difícil sustraernos a ellade mirar el mundo y de mirarnos a nosotros mismos. Hablar de las Malvinas no es solamente hablar de la relación entre el archipiélago y la nación, sino primordialmente de nuestro nacionalismo» ${ }^{21}$.

\section{La Guerra de Malvinas}

Quizás el hecho más trascendental del conflicto fue la Guerra de Malvinas. Luego del desembarco de tropas argentinas el 2 de Abril de 1982, comenzó la guerra que dejó consigo numerosas muertes de

19 Documento Soberanía Argentina en Malvinas. A 50 años del "Alegato Ruda» (2014), Buenos Aires, Ministerio de Relaciones Exteriores. Pág. 15.

${ }^{20}$ Cfr. Considerandos Resolución 3160 de la Asamblea General de la Organización de las Naciones Unidas.

${ }^{21}$ Cfr. PALERMO, Vicente (2007). Sal en las heridas. Las Malvinas en la cultura argentina contemporánea, Buenos Aires, Ed. Sudamericana. Págs. 16-17. 
militares argentinos y que duraría hasta el 14 de Junio, día en que Argentina firmó su rendición.

Esta guerra no sólo dejó un hueco vacío en las familias de quienes dieron su vida por la causa soberana ${ }^{22}$, sino que agudizó el enfrentamiento entre los dos países.

Las consecuencias obvias de las pérdidas materiales y, más aún, de vidas humanas, dieron muestra de la hostilidad de las relaciones y de la necesidad de dialogar sobre el reclamo. Además, el cese del fuego llevó a que Argentina dejara atrás una etapa oscura de gobiernos de facto para volver a encauzarse en la democracia y a que el gobierno inglés intensificara su presencia en las Islas, otorgando la ciudadanía británica plena a los habitantes y generando una serie de medidas económicas que tendieron a que se fortaleciera este sector que había sido olvidado durante muchos años por la Corona.

Si bien derrotada militarmente, el alma mater que llevó a la guerra -la defensa de la soberanía argentina- siguió presente en el pueblo y los gobiernos radicales y peronistas que se sucedieron el poder. Fue el sentir propio de la nación, del pueblo, lo que llevó al Convencional Constituyente reformador de 1994 a establecer una Disposición Transitoria -la primera-, en la que se lee: «la Nación Argentina ratifica su legítima e imprescriptible soberanía sobre las Islas Malvinas, Georgias del Sur y Sandwich del Sur y los espacios maritimos e insulares correspondientes, por ser parte integrante del territorio nacional. La recuperación de dichos territorios y el ejercicio pleno de la soberanía, respetando el modo de vida de sus habitantes, y conforme a los principios del derecho internacional, constituyen un objetivo permanente e irrenunciable del pueblo argentino» ${ }^{23}$. Esta disposición es un mandato a todos los argentinos, y no por nada es transitoria, porque el pueblo augura que la historia mostrará su recuperación en algún momento.

\section{LOS OCUPADORES DE LAS ISLAS}

Ya comprendiendo el esquema general sobre el que se circunscriben las disputas sobre el caso, deberemos adentrarnos en el eje del problema que llevó a encauzar a 30 años de la guerra a un nuevo enfrentamiento diplomático. Por ello, es necesario comprender en primer lugar cuál es la naturaleza que ostentan los pobladores de las Islas, sobre los cuales pesará el conflicto que trataremos de analizar.

\footnotetext{
22 Argentina perdió más de 600 hombres en la guerra.

${ }^{23}$ Cfr. Constitución de la Nación Argentina, Disposición Transitoria Primera.
} 


\section{A. Los kelpers}

Quienes habitan las Islas Malvinas se denominan tradicionalmente como «kelpers» y guarda el nombre su razón en que las grandes algas marinas que rodean las Islas se llaman «kelp». Otras denominaciones para estos habitantes son «islanders», «falkland islanders» o «falklanders».

El término «kelper» ha sido usado peyorativamente por Argentina para tratar a los habitantes de las Islas Malvinas, quizás de manera despectiva ante su ilegítima ocupación. Señala Alejandro Betts que "un kelper es un malvinense nativo de las islas; es un colono que representa la ocupación física británica de un espacio territorial que no corresponde a la Corona Británica. Hasta 1983, el malvinense nativo no tenía ningún documento de identidad nacional, ni como británico, ni como argentino. Éramos indocumentados y huérfanos de Patria» ${ }^{24}$.

Quienes ocupan las Islas se consideran británicos, aunque con una identidad particular, con una mentalidad que es más proclive al pensamiento de la Corona que al de la República. Su distancia con Gran Bretaña ayuda a una identidad propia de los kelpers, quienes son una nación de inmigrantes implantados en las islas, que ya han visto nacer a varias generaciones de personas en el archipiélago. Algunos de los kelpers consideran que su mayor aporte a Gran Bretaña es la ocupación que realizan de las Islas de manera permanente, lo que ayuda a que éste país mantenga su título de posesión ininterrumpida de las éstas. La historia que se cuenta en las escuelas a los kelpers niega los derechos argentinos y desconoce facetas del devenir temporal de las Islas ${ }^{25}$.

\section{B. El gobierno de las Islas Malvinas (Falkland Islands)}

Los kelpers encuentran un orden de gobierno propio para las Islas Malvinas (Falkland Islands), que está desarrollado en la «Constitución del territorio Británico de Ultramar de las Falkland Islands» ${ }^{26}$ de 2008, que reconoce como Jefe de Estado a la Reina de Inglaterra, Isabel II.

${ }^{24}$ Se puede consultar la conferencia brindada el 4 de Febrero de 2012 por Alejandro Betts, un malvinense de cuarta generación en http://www.nuestromar.org/ noticias/05-02-12/\%E2\%80\%9Cpensar-malvinas\%E2\%80\%9D-0.

${ }^{25}$ Se puede consultar la nota "En las Malvinas sólo se enseña una versión de la historia» de Nicolás Ballinotti en http://www.lanacion.com.ar/1462555-en-las-malvinas-solo-se-ensena-una-version-de-la-historia.

${ }^{26}$ Se puede consultar en http://www.falklands.gov.fk/assets/The-Falkland-IslandsConstitution-Order-2008.pdf. 
Cuenta con un Gobernador de las Islas ${ }^{27}$, quien actúa en nombre de la Corona $^{28}$ y tiene el poder de ésta en su ausencia territorial. Existe un Consejo Ejecutivo de las Islas ${ }^{29}$, con poderes ejecutivos para asesorar al Gobernador. La administración pública de las Islas cuenta con un Jefe Ejecutivo ${ }^{30}$, quien es responsable del gobierno del archipiélago.

El poder legislativo de las Islas reside en la Asamblea ${ }^{31}$, que reemplazó al Consejo Legislativo luego de la Constitución de 2008, y que debe dictar leyes para la paz, el orden y el buen gobierno. La Asamblea es unicameral y tiene ocho miembros electos, dos ex officio (el Jefe del Ejecutivo y el Director de Finanza) y el Presidente.

Esta forma de gobierno, impulsada por la Constitución de 2008, lleva consigo la posibilidad de tener ciertas competencias necesarias para asumir tareas de gobierno y de consulta al pueblo. El entendimiento de las instituciones de las Islas nos lleva a comprender cómo se encauzó el referéndum del año 2013 en esa porción austral.

\section{EL PRETENDIDO DERECHO DE AUTODETERMINACIÓN DE LOS KELPERS Y EL REFERÉNDUM DE 2013}

\section{A. Nación, pueblo y autodeterminación}

1. ¿Son los kelpers una nación?

Una de las primeras teorías que surge en relación a los kelpers es que constituirían una nación dentro del esquema británico. Algunos autores sostienen que "no hay, en el Reino Unido, una nación titular de la soberanía (...). En consecuencia, al carecer alli el término nación de su preciso significado jurídico-constitucional, y tener únicamente el más vago y flexible significado cultural, sociológico o politológico, no habría reparo alguno para entender el Estado británico como un Estado plurinacional, en el sentido descriptivo de Estado que integra a diversos pueblos o comunidades (que, obviamente, carecen de soberanía)„32. Así, al no

${ }^{27}$ En inglés, the Governor.

${ }^{28}$ Según el artículo 56.2. de la Constitución: «subject to this Constitution, the executive authority of the Falkland Islands shall be exercised on behalf of Her Majesty by the Governor, either directly or through officers subordinate to him or her».

${ }^{29}$ En inglés, The Executive Council of the Falkland Islands.

${ }^{30}$ En inglés, The Chief Executive of the Falkland Islands.

${ }^{31}$ En inglés, Legislative Assembly of the Falkland Islands.

32 Cfr. ARAGÓN REYES, Manuel (2014). «Problemas del Estado Autonómico» en Asamblea, Revista Parlamentaria de la Asamblea de Madrid N. ${ }^{\circ}$ 31, Diciembre 2014, Madrid, Servicio de Publicaciones de la Asamblea de Madrid. 
existir una sola nación en la que se deposite la soberanía, deberíamos preguntarnos si los kelpers se constituirían en una nacionalidad dentro de la Corona Británica, lo que nos lleva a tratar de comprender si ese carácter nacional podría devenir en una independencia de los isleños.

Antes que nada, señalamos con Andrés de Blas que el nacionalismo «es una realidad difícil de aprehender en el marco de una teoría explicativa de carácter global» ${ }^{33}$, por lo que quizás el entendimiento que realicemos a continuación no es abarcativo de todas las realidades que se suscriben a éste. Estos nacionalismos "pueden generar teorías y movimientos sociales que destacan por su especial conflictividad social» ${ }^{34}$.

Es imprescindible realizar una distinción entre nacionalismo político y cultural. La primera idea tiene que ver con «un mayor sentido funcional y pragmático, en tanto que fuente de legitimidad y generador de lealtad hacia una forma de Estado-nación que, en el mundo occidental, se ha terminado transformando en una realidad equiparable al sistema político liberal-democrático» ${ }^{35}$. La segunda idea, de base alemana, tiende a "acentuar rasgos más emotivos y comprometidos, menos acordes con una lógica instrumental, en coherencia todo ello con el modo de concebir la nación como objetivo en sí mejor que como artefacto al servicio de la vida politica» ${ }^{36}$. Sobre la idea de nación cultural se sostiene el principio de las nacionalidades, por lo que "alli donde no se ha conseguido reconciliar al Estado con la nación cultural, se consideraría lógico que las nacionalidades, entendidas como naciones no realizadas politicamente, reclamen el acceso a su propia estatalidad» ${ }^{37}$.

En las cuestiones nacionalistas hay manifestaciones que tienen que ver con identidades étnico-lingüísticas «con aspiraciones políticas enfrentadas a los Estados de que forman parte» ${ }^{38}$. En los expedientes nacionales tienen una suma importancia las realidades lingüísticas, cuestión que ocupa un lugar trascendental, ya que "de todos los elementos culturales que intervienen en la génesis, desarrollo y transformación de los movimientos e ideologías nacionalistas, ninguno ha alcanzado la importancia de la lengua» ${ }^{39}$. El hecho de tener una lengua es un factor cohesionante de la sociedad que se proclama nación, y le

${ }^{33}$ Cfr. DE BLAS, Andrés (1994). Nacionalismos y naciones en Europa, Madrid, Alianza Universidad, Pág. 16.

${ }^{34}$ Cfr. RUÍZ RODRÍGUEZ, Segundo (1998). La teoría del derecho de autodeterminación de los pueblos, Madrid, CEPC. Pág. 27.

${ }^{35}$ Cfr. DE BLAS, Andrés (1994). Op. cit. Pág. 16.

36 Idem.

37 Cfr. DE BLAS, Andrés (1994). Op. cit. Pág. 125.

${ }^{38}$ Cfr. DE BLAS, Andrés (1994). Op. cit. Pág. 33.

39 Cfr. DE BLAS, Andrés (1994). Op. cit. Pág. 101. 
atribuye una singularidad cultural de notorio nivel, que genera una distinción entre ese pueblo y otros que hablan otras lenguas. Otro factor trascendente es el religioso, que imprime en la nación no sólo una forma de entender la deidad, sino un parámetro moral de conductas y de asimilación de la realidad. La historia y la geografía que ocupan los nacionalismos son puntos esenciales a tener en cuenta para poder definir su contorno. En fin, un nacionalismo tiene que "potenciar y poner de manifiesto aquellos rasgos culturales, fisiológicos e históricos de los que pueden resultar elementos de diferenciación» ${ }^{40}$.

Frente a esa descripción, podemos decir que los kelpers no se constituyen en una nación cultural diferenciada de la generalidad británica, sobre todo teniendo en cuenta que no hay rasgos que permitan apreciar una peculiaridad tal que lleve a estos pobladores a querer separarse de Gran Bretaña, cuestión que se demuestra en que incluso uno de los mayores reclamos que se han realizado tienen que ver con la cuestión de la adquisición de ciudadanía plena.

Los kelpers han vivido cambios profundos en los últimos 30 años, pasando de ser ciudadanos de segunda a ciudadanos plenos de Gran Bretaña, quizás sustentándose el cambio en la necesidad de la metrópoli de contar con la adhesión no sólo en los sentimientos sino también en el derecho de estos pobladores.

Uno de los rasgos esenciales de las naciones, como lo es el de la lengua, no aparece diferenciado en ese sector del archipiélago. La lengua inglesa, con pronunciación británica es una de las pautas que muestran su mayor identidad con la Corona.

Todo ello nos lleva a pensar que los kelpers no son una nación cultural, y no pesa sobre ellos una diferenciación pronunciada del resto de los habitantes de Gran Bretaña que les dé el derecho a poder pensar en una independencia y en la construcción de un Estado propio, sobre todo por ser legalmente ciudadanos ingleses y, además, profesar sus distintivos rasgos culturales.

2. ¿Pueden los kelpers autodeterminarse como pueblo?

Si no son una nación cultural, podríamos pensar que los kelpers se constituyen en aquello que la Comunidad Internacional ha denominado como "pueblo», característica que les daría la posibilidad de ejercer el derecho de autodeterminación, premisa que tuvieron en los últimos años.

${ }^{40}$ Cfr. RUÍZ RODRÍGUEZ, Segundo (1998). Op. cit. Pág. 28. 
Las ideas de gobierno representativo hacen que se asuma la teorización de principios que tienen que ver con la autodeterminación, que defiende "que los ciudadanos deben elegir su gobierno de modo que éste repose sobre su consentimiento; igualmente, que puesto que los hombres son libres y racionales, deben participar en la vida de aquél» ${ }^{41}$. Este derecho de autodeterminación tiene que ver con la posibilidad de los pueblos de establecer libremente su condición política, proveyéndose de su propio desarrollo cultural, económico y social. Este derecho "ha ocupado todo el espacio que, en el Siglo XIX y la primera mitad del siglo $X X$, compartía con ese principio (de las nacionalidades)» ${ }^{42}$.

Juan José Solozábal se refiere a este derecho identificándolo como "la decisión soberana en un solo acto de una comunidad territorial sobre su propia forma politica, manifestando su voluntad de separarse o mantener su integración en el Estado, con su actual posición u otra diferente» ${ }^{43}$.

La Carta de las Naciones Unidas de 1945 habla sobre el derecho de autodeterminación, cuestión ampliada en el Documento explicativo, entendiendo que se refiere a la auto administración, mas no a una secesión. Asimismo, el Pacto Internacional de Derechos Civiles y Políticos y el Pacto Internacional de Derechos Económicos, Sociales y Culturales, hacen mención de la autodeterminación, entendiendo así la posibilidad de establecer su libre condición política y proveer a su desarrollo económico, social y cultural.

De la interpretación de estos textos se extrae que "no supone el reconocimiento de la secesión salvo en las situaciones coloniales y en los supuestos de que ésta se pretenda en un Estado racista o que no represente la totalidad del territorio ${ }^{44}$. Así, si quisiéramos diferenciar el derecho de autodeterminación del principio de las nacionalidades podemos hacerlo "en el sentido de que el primero no exige necesariamente la secesión, lo que sí ocurre si se pretende aplicar el principio de las nacionalidades» ${ }^{45}$, aunque cabe destacar que "pese a que el 'derecho

41 Cfr. DE BLAS, Andrés (1994). Op. cit. Pág. 144.

${ }^{42} C f r$. DE BLAS, Andrés (2014). "Sobre el principio de las nacionalidades» en SOLOZÁBAL, Juan José (2014). La autodeterminación a debate, Madrid, Editorial Pablo Iglesias. Pág. 55.

${ }^{43}$ Cfr. SOLOZÁBAL, Juan José (2014). La autodeterminación a debate, Madrid, Editorial Pablo Iglesias. Pág. 200.

${ }^{44}$ Cfr. RUIZ RODRÍGUEZ, Segundo (1998). Op. cit. Pág. 75.

${ }^{4}$ Cfr. RUIZ RODRÍGUEZ, Segundo (1998). Op. cit. Pág. 138. 
de autodeterminación' no exige la secesión, sí la incluye entre las posibilidades que de su aplicación se pueden derivar» ${ }^{46}$.

Por esto, ¿cabe la posibilidad de los pobladores de las Islas a «autodeterminarse»? Entendemos que no pueden hacerlo, poniendo la situación a la luz del derecho internacional y la interpretación doctrinal vigente en relación a este derecho.

\section{B. El referéndum de 2013}

\section{El meollo del asunto}

Durante la primer década del siglo XXI, comienzan a hacerse eco en la comunidad internacional de una serie de actitudes de los pobladores de las Islas y de su gobierno de limitar las posibilidades de asentamiento de argentinos en ese espacio, sobre todo poniendo trabas burocráticas a la adquisición de propiedades, de manera que se impedía el posible asentamiento de personas de este país. Esta situación no era novedosa, ya que quienes estaban a cargo del gobierno de las Islas mantenían esta política desde antaño, como un claro símbolo de hegemonía sobre el territorio.

La proximidad a los 30 años de la guerra quizás fue un reflotamiento de las actividades diplomáticas que llevaron a que existiese una disputa de ideas entre las agendas internacionales de ambos países.

Las posiciones argentinas acerca de la ocupación ilegal de las Islas y sobre el status de la población implantada en ese territorio hicieron que el eco de las palabras llegase a la Gobernación de las Falkland Islands. Así, una de las posibilidades de generar una baja de la imagen negativa que existía sobre los kelpers era que éstos se expresaran al respecto. Argentina mantenía firme suposición de que los habitantes de las Islas responden a la Corona y que son parte de un proceso colonizador.

El Gobernador Haywood se planteó, entonces, llevar a cabo un referéndum para que se expresase un supuesto derecho de autodeterminación de los kelpers, en el que indicarían si querían seguir formando parte de Gran Bretaña o no. De esta manera, expresaba su parecer diciendo que este camino era necesario para responder a los reclamos argentinos, de tal manera que llega a comprender -lo que

${ }^{46}$ Cfr. RUIZ RODRÍGUEZ, Segundo (1998). Op. cit. Pág. 139. 
proclamó con sus palabras- que llevándolo a cabo: «we can solve the issue once and for all» ${ }^{47}$.

Así, «el 12 de junio de 2012, el 'gobierno' de las Islas Malvinas (Falkland Islands Government, sic) anunció su intención de celebrar un referéndum, en orden a permitir a los isleños expresar su opinión sobre la condición política de las Islas» ${ }^{48}$.

La Asamblea estableció una Ordenanza ${ }^{49}$ que se aprobó el 31 de Octubre de 2012 y que se publicó el 2 de Noviembre del mismo año, y el Gobernador estableció en una Orden del 20 de Diciembre la pregunta que se realizaría, la que luego de un preámbulo ${ }^{50}$, se configuraba de la siguiente manera: «do you wish the Falkland Islands to retain their current political status as an Overseas Territory of the United Kingdom?».

Básicamente, se buscaba en este referéndum un supuesto «derecho de autodeterminación», a través de la expresión popular sobre el caso. Este aludido derecho se expresaba claramente en el preámbulo, al decir: "Under the Falkland Islands Constitution the people of the Falkland Islands have the right to selfdetermination».

La fecha en la que se iba a realizar el referéndum era en los días 10 y 11 de Marzo de 2013. Dos días antes de producirse, la Cancillería Argentina expresó una nota de prensa $^{51}$ en la cual indicaba que "el Reino Unido ha convocado a una votación de los pobladores que ese país implantó en las Islas Malvinas para preguntarles sobre cuestiones que pretenden tergiversar la verdadera condición jurídica en que se encuentran esas islas» ${ }^{52}$. Se sostiene en el comunicado la defensa de la posición argentina y de la comunidad internacional al respec-

47 Se recomienda consultar http://en.mercopress.com/2012/03/29/falklands-opento-un-referendum-to-decide-whether-islanders-want-to-remain-british.

${ }^{48}$ Cfr. PEZZANO, Luciano (2013). Op. cit. Pág. 3.

${ }^{49} \mathrm{La}$ que puede consultarse en http://www.falklands.gov.fk/assets/ReferendumFalkland-Islands-Political-Status-Ordinance-2012.pdf.

${ }_{50}$ Que decía: "The current political status of the Falkland Islands is that they are an Overseas Territory of the United Kingdom. The Islands are internally selfgoverning, with the United Kingdom being responsible for matters including defence and foreign affairs. Under the Falkland Islands Constitution the people of the Falkland Islands have the right to selfdetermination, which they can exercise at any time. Given that Argentina is calling for negotiations over the sovereignty of the Falkland Islands, this referendum is being undertaken to consult the people regarding their views on the political status of the Falkland Islands. Should the majority of votes cast be against the current status, the Falkland Islands Government will undertake necessary consultation and preparatory work in order to conduct a further referendum on alternative options».

51 Disponible en http://www.cancilleria.gov.ar/es/un-intento-britanico-mas-demanipular-la-cuestion-malvinas.

52 Consultar la página mencionada en la nota precedente. 
to de las Islas, cuestión que genera el sostenimiento de la idea de que Gran Bretaña se ubica «en una actitud claramente demostrativa de la falta de fundamentos para sus pretensiones y sobre todo carente de buena $f e »^{53}$.

\section{El desarrollo del referéndum}

Finalmente, el referéndum se llevó a cabo en la fecha prevista. Según el Gobierno de las Islas, participaron 1.518 votantes, que representan un $92 \%$ del padrón. La votación arrojó como resultados:

-1 voto rechazado;

- 1.513 votos fueron por el «Sí», lo que representa el 99,8\%;

$-3 \operatorname{votos}(0,2 \%)$, fueron por el «No», lo que representa el 0,2\%; y

-1 voto no fue considerado.

\section{La validez del referéndum. El rechazo argentino}

Ahora bien, es necesario analizar si el referéndum que se utilizó es un canal correcto para poder encauzar el pretendido derecho de autodeterminación. Así, señala Pezzano que "para que un referéndum pueda considerarse como una forma válida de ejercicio de la libre determinación, deben reunirse tres requisitos fundamentales, que surgen de la práctica de la Organización: 1) El sujeto consultado debe ser un pueblo, titular del derecho a la libre determinación; 2) No puede ser contrario al derecho internacional vigente; y 3) Debe ser organizado de manera tal que asegure la participación y la libre expresión de la voluntad del pueblo de que se trata» ${ }^{54}$. Pero para que una población sea realmente considerada un pueblo que pueda dar lugar a la autodeterminación, se la tiene que comprender como «una entidad social que posee una identidad clara y características propias que lo distinguen, y que a la vez tiene una particular relación con un territorio, es decir, que está constituido por la población autóctona u originaria de ese territorio» ${ }^{55}$.

Visto de esta manera, consideramos que la forma en que se encauzó el referéndum para hacer valer el derecho de autodeterminación, es una manifestación equivocada al respecto y carece de validez como

\footnotetext{
${ }^{53}$ Idem.

${ }^{54}$ Cfr. PEZZANO, Luciano (2013). Op. cit. Pág. 6.

55 Idem.
} 
un título que pueda ser sostenido por Gran Bretaña para continuar haciendo un uso ilegítimo de la posesión isleña. Por ello, "cualquier consulta que pueda efectuarse a la población de Malvinas no tendrá ningún valor como forma ejercicio de la libre determinación, al no existir ningún titular de este derecho» ${ }^{56}$.

La Cancillería Argentina, en uso de la representación internacional que ostenta, ha tratado de enunciar sus argumentos en relación con el referéndum, lo que básicamente se suscribe a dos líneas argumentativas:

- La primera, que sostiene que el pueblo de las Islas no es nativo, sino que ha sido implantado por Gran Bretaña, más allá de la posibilidad de aducir que existan generaciones de pobladores. Sobre esto, la Asamblea General de la ONU ha explicado que «la libre determinación es básicamente un derecho de la población autóctona» ${ }^{57}$. Asimismo, se ha afirmado que «el ejercicio del derecho a la libre determinación sólo corresponde a los pueblos autóctonos y con personalidad inconfundible y no a las poblaciones adventicias oriundas de la potencia colonial, afines a la misma o a su servicio» ${ }^{58}$.

Esta primer línea, sumada a la identidad de los kelpers con Gran Bretaña -más allá de su autogobierno- es un punto esencial en la manifestación de que no es posible una autodeterminación;

- La segunda, que se sostiene en el entendimiento argentina de que este recurso que quiere ser utilizado por Gran Bretaña, trata de soslayar las vías diplomáticas de discusión acerca de la soberanía de las Islas con Argentina. Asimismo, subrayando esta actitud, Argentina denunció de Gran Bretaña que la "falaz invocación de este principio en esta Cuestión contrasta con su posición en otros casos de descolonización, tal como el del archipiélago de Chagos, en el cual expulsó a sus habitantes nativos, privándolos, al día de hoy, de su derecho a retornar a sus tierras. Tampoco recabó el Gobierno británico la opinión de

${ }^{56}$ Cfr. PEZZANO, Luciano (2013). Op. cit. Pág. 26.

57 Cfr. DRNAS DE CLÉMENT, Zlata: «El Derecho de libre determinación de los pueblos. Colonialismo formal. Neocolonialismo. Colonialismo interno». Anuario Argentino de Derecho Internacional III, 1987-1989, Córdoba. Pág. 213.

${ }^{8} C f r$. INSTITUTO HISPANO LUSO AMERICANO DE DERECHO INTERNACIONAL (1977), «Resolución sobre el Derecho de autodeterminación de los pueblos y su campo de aplicación» (Ponente: Antonio GOMEZ ROBLEDO), en Resoluciones adoptadas en el XI Congreso, Madrid-Salamanca, Disponible en: http://www.ihladi.org/ resoluciones_XI.pdf. 
los habitantes de Hong Kong cuando restituyó dicho territorio a su legítimo titular, la República Popular China» ${ }^{59}$.

\section{CONCLUSIONES}

Frente a todo lo expuesto, debemos considerar que la cuestión Malvinas sigue siendo uno de los grandes problemas diplomáticos argentinos. Aún así, la data de comienzo en 1833 del reclamo soberano ininterrumpido por parte de las autoridades del país austral son un fundamento claro para destacar el compromiso evidenciado por Argentina en sostener que es el legítimo propietario de las Islas.

Más allá de que no se haya logrado una solución ni por medios diplomáticos ni bélicos para la cuestión, la Comunidad Internacional encuentra un papel destacable en todo el esquema de relaciones que se quieren encauzar para que la solución sea pacífica y para que ésta esperada solución ponga cese al proceso colonialista que la Corona sostiene sobre las Islas.

Ahora bien, las Islas no están solas y están ocupadas por ciudadanos británicos que, bajo el manto de querer ostentar características propias a las de una nacionalidad que no son, mantienen la posesión de éstas por parte de Inglaterra. Esta situación no puede ser desconocida y es uno de los mayores problemas que se afrontan en la actualidad por el caso Malvinas, porque su identidad británica ha hecho que se sostengan generaciones de colonos en esas frías tierras y son una deuda pendiente en los diálogos soberanos al respecto.

Hemos afirmado que no es posible dar lugar a una secesión basada en el principio de la nacionalidad ni mucho menos en el expediente extraordinario de la autodeterminación, por no tener los falkers la entidad de "pueblo» conforme la interpretación que la Comunidad Internacional ha requerido para poder hacer uso de éste.

Por otro lado, la canalización del pretendido derecho de autodeterminación a través del referéndum que el gobierno de las Islas ha querido llevar a cabo no sólo que no es sustantivamente correcto, sino que además irrumpe en contra de la Resolución 31/49 de la Asamblea General de la ONU, que sostuvo que tanto Inglaterra como Argentina deben abstenerse «de adoptar decisiones que entrañen la introducción

${ }^{59} C f r$. http://www.cancilleria.gov.ar/es/un-intento-britanico-mas-de-manipular-lacuestion-malvinas. 
de modificaciones unilaterales en la situación mientras las Islas están atravesando por el proceso recomendado» ${ }^{60}$.

No podemos dejar de destacar una cuestión que nos parece central en orden al futuro de las relaciones diplomáticas, sobre todo en el caso de que Inglaterra acepte que las Malvinas son argentinas, que es el de cómo gestionar un espacio con pobladores que hablan otra lengua, con una cultura absolutamente distinta y con una identificación clara a la metrópoli. Por ello, en el caso de reabrir las discusiones entre los dos países, éstas tienen que dar un paso más hacia adelante. No basta sólo con presentar los títulos válidos que Argentina sostiene, sino que se requiere, además, una propuesta de integración del pueblo malvinense a la República Argentina, que no dé lugar a dudas acerca de una posible segregación o discriminación de ese sector poblacional. Somos conscientes de que los pobladores de las Islas no guardan el mayor agrado de los argentinos, pero esa actitud debería cambiar a una más conciliadora en miras a lograr, además de la victoria del derecho, la vinculación popular.

Bregamos en estas líneas por mostrar un esquema general que pretende comprender la naturaleza del conflicto y la disputa actual en torno a las cuestiones poblacionales que presenta. Convocamos, desde esta tribuna, a un comportamiento serio de relaciones diplomáticas que tiendan en el Siglo XXI -en el marco del Estado de derecho- a lograr la paz y la descolonización de territorios ilegítimamente ocupados.

\section{BIBLIOGRAFÍA Y FUENTES DOCUMENTALES}

\section{Libros y artículos}

ARAGÓN REYES, Manuel (2014). «Problemas del Estado Autonómico» en Asamblea, Revista Parlamentaria de la Asamblea de Madrid N. ${ }^{\circ}$ 31, Diciembre 2014, Madrid, Servicio de Publicaciones de la Asamblea de Madrid.

DE BLAS, Andrés (1994). Nacionalismos y naciones en Europa, Madrid, Alianza Universidad.

- (2014). «Sobre el principio de las nacionalidades» en SOLOZÁBAL, Juan José (2014). La autodeterminación a debate, Madrid, Editorial Pablo Iglesias. Unidas.

${ }^{60}$ Cfr. Resolución 31/49, Asamblea General de la Organización de las Naciones 
DESTEFANI, Laurio H. (1982). Malvinas, Georgias y Sandwich del Sur, ante el conflicto con Gran Bretaña, Buenos Aires, Edipress S. A.

DÍAZ ALEJO, Raimundo (1982). «El problema de las Malvinas» en Quinto Centenario N. ${ }^{\circ}$ 4, Madrid UCM. Págs. 175-208.

DRNAS DE CLÉMENT, Zlata: «El Derecho de libre determinación de los pueblos. Colonialismo formal. Neocolonialismo. Colonialismo interno». Anuario Argentino de Derecho Internacional III, 1987-1989, Córdoba.

HOPE, Adrián F. J. (1983). «Soberanía y descolonización de las Islas Malvinas (Falkland Islands)» en Boston College International and comparative law review Vol. 6, N. ${ }^{\circ}$ 2. Boston, Boston College.

INSTITUTO HISPANO LUSO AMERICANO DE DERECHO INTERNACIONAL (1977). «Resolución sobre el Derecho de autodeterminación de los pueblos y su campo de aplicación» (Ponente: Antonio GOMEZ ROBLEDO), en Resoluciones adoptadas en el XI Congreso, Madrid-Salamanca.

KOUTOUDJIAN, Adolfo y otros (2001). Geopolítica y Globalización. Estado-Nación, autodeterminación, región y fragmentación, Buenos Aires, Eudeba.

PALERMO, Vicente (2007). Sal en las heridas. Las Malvinas en la cultura argentina contemporánea, Buenos Aires, Ed. Sudamericana.

RUÍZ RODRÍGUEZ, Segundo (1998). La teoría del derecho de autodeterminación de los pueblos, Madrid, CEPC.

SOLOZÁBAL, Juan José (2014). La autodeterminación a debate, Madrid, Editorial Pablo Iglesias.

VILARÓ, Ramón (2007). «En Malvinas huele a petróleo» en Revista Cambio 16, N. ${ }^{\circ} 1845$ (ABR 17), Madrid.

\section{Documentos y Resoluciones de Organismos Nacionales e Internacionales}

Constitución de la Nación Argentina.

Constitución del territorio Británico de Ultramar de las Falkland Islands.

Documento Falkland Islands Census 2012: headline results.

Documento Soberanía Argentina en Malvinas. A 50 años del «Alegato Ruda». 
Resolución 31/49 de la Asamblea General de la Organización de las Naciones Unidas.

Resolución 1514 de la Asamblea General de la Organización de las Naciones Unidas.

Resolución 2065 de la Asamblea General de la Organización de las Naciones Unidas.

Resolución 3160 de la Asamblea General de la Organización de las Naciones Unidas. 